\title{
BMJ open Qualitative cross-sectional study of the perceived causes of depression in South Asian origin women in Toronto
}

\author{
Samanthika Ekanayake, ${ }^{1}$ Farah Ahmad, ${ }^{2}$ Kwame McKenzie ${ }^{1,3}$
}

\begin{abstract}
To cite: Ekanayake S, Ahmad F, McKenzie K. Qualitative cross-sectional study of the perceived causes of depression in South Asian origin women in Toronto. BMJ Open 2012;2:e000641. doi:10.1136/

bmjopen-2011-000641

- Prepublication history for this paper is available online. To view these files please visit the journal online (http:// dx.doi.org/10.1136/ bmjopen-2011-000641)
\end{abstract}

Received 17 November 2011 Accepted 24 January 2012

This final article is available for use under the terms of the Creative Commons Attribution Non-Commercial 2.0 Licence; see http://bmjopen.bmj.com

\section{${ }^{1}$ Social Aetiology of Mental IIIness (SAMI) Training Program, Health Systems and Health Equity Research Group, Centre for Addiction and Mental Health (CAMH), Toronto, Ontario, Canada ${ }^{2}$ School of Health Policy and Management, Faculty of Health, York University, Toronto, Ontario, Canada ${ }^{3}$ Department of Psychiatry, University of Toronto, Toronto, Ontario, Canada}

Correspondence to Professor Kwame McKenzie; kwame_mckenzie@camh.net

\section{ABSTRACT}

Objective: To explore how South Asian origin women in Toronto, Canada, understand and explain the causes of their depression.

Design: Cross-sectional in-depth qualitative interviews.

Setting: Outpatient service in Toronto, Ontario.

Participants: Ten women with symptoms of depression aged between 22 and 65 years of age. Seven were from India, two from Sri Lanka and one from Pakistan. Four were Muslim, three Hindu and three Catholic. Two participants had university degrees, one a high school diploma and seven had completed less than a high school education. Eight were married, one was unmarried and one a widow.

Results: Three main factors emerged from the participant narratives as the causes of depression: family and relationships, culture and migration and socioeconomic. The majority of the participants identified domestic abuse, marital problems and interpersonal problems in the family as the cause of their depression. Culture and migration and socioeconomic factors were considered contributory. None of our study participants reported spiritual, supernatural or religious factors as causes of depression.

Conclusion: A personal-social-cultural model emerged as the aetiological paradigm for depression. Given the perceived causation, psycho-social treatment methods may be more acceptable for South Asian origin women.

\section{INTRODUCTION}

The way that people understand and explain illness depends on many factors including cultural background, education level, health beliefs, attitudes and knowledge and trust in healthcare systems. ${ }^{1-3}$

Illness explanatory models (IEM) examine the perspectives people have of illness, mainly focusing on aetiology, symptoms, severity, prognosis, reasons for consultation and treatment preferences. ${ }^{4}$ Research suggests that IEM ultimately determine a number of factors including help-seeking behaviour, treatment compliance, satisfaction, selection

\section{ARTICLE SUMMARY}

\section{Article focus}

- An exploration of the perceived causes of depression in women of South Asian origin in Toronto, Canada.

Key messages

- Depression in South Asian women in Toronto may be caused by social problems that could be the target for prevention and health promotion.

- Given the perceived causation, psycho-social interventions may be more acceptable for South Asian origin women in Toronto.

- Links between social and health services may be important in decreasing the burden of depression in South Asian origin women in Toronto.

Strengths and limitations of this study

- This study was able to interview a diverse crosssection of South Asian origin women in a community setting.

- The interviewer was also a South Asian women and this may have facilitated disclosure.

- The study did not disaggregate the South Asian group into different religious groups or countries of origin.

- This study only included participants who could speak English.

of pathways to care and selection of treatment. $^{5}{ }^{6}$ IEM may vary between- and within-ethnic groups, gender groups and different generations as well as for different types of illness. ${ }^{7-9}$ A better understanding of conceptual models of illness may help to improve service delivery and clinical outcomes and reduce healthcare cost. ${ }^{10}$

Depression is one of the most underrecognised and under-treated mental illnesses in primary care globally. ${ }^{11}$ It is one of the most common and costly mental health problems in Canada. ${ }^{12} 13$

The rates of depression in immigrants in Canada vary; some groups such as older adults and some South and East Asian groups may be at increased risk. ${ }^{14}$ Though the use of general medical care is similar for 
immigrants and non-immigrants but immigrants are less likely to use mental health services. ${ }^{15} 16$ The reasons for the increased rates of depression have not been well documented in Canada but with regards to service use studies report that biomedical models of health systems may act as barriers to care by conflicting with the views of patients from other traditions. ${ }^{17-19}$

Theoretical models of the aetiology of depression are diverse. ${ }^{20}$ Biomedical models consist of genetic, physical or somatic causation. Socio-cultural models include life circumstances and cultural factors, while psychological models focus on psychological or behavioural factors. A multiple causation, bio-psycho-social, model has been widely accepted. ${ }^{21-23}$

The lay understanding of depression varies between cultural and ethnic groups. For instance in European countries, the majority of patients endorse bio-psychiatric models, while in East and South East Asia, psychosocial models predominate. ${ }^{24}$ Some argue that this may be linked to differences in help seeking as the former prefer psychiatric treatment, ${ }^{8}$ while the latter prefer more traditional socially focused healing modalities. ${ }^{14} 19$

Though it should be noted that patients may not seek help when there is clearly a need. ${ }^{19}$

Among the few Canadian studies that are focused on conceptual understandings of depression, Schreiber and Hatrick $^{25}$ reported that Euro-North American women predominantly report a biomedical explanatory models. But, to our knowledge, there are no studies conducted in Canada that have investigated the conceptual models of depression in minority ethnic groups. In order to begin to fill this gap in the literature, we conducted a qualitative study of women of South Asian origin. The aim of the study was to identify and document how South Asian origin women in Toronto, Canada, understand and explain the causes of their depression. This was an initial study to test the feasibility of recruitment to see whether South Asian women with depression would speak with researchers about causation and to map out the range of models of causation in population to aid the development of questionnaires and hypotheses for possible larger mixed methods studies.

The South Asian population is the largest visible minority in Canada. ${ }^{26}$ South Asian refers to people who originate from India, Sri Lanka, Bangladesh, Pakistan and Nepal. They are a diverse group with significant ethnic, religious, and linguistic differences, but it has been argued that these groups share commonalities in their social networks, family interactions and customs and traditions. ${ }^{14} 27$ This study investigated only one gender groups because conceptual models of depression vary by gender. ${ }^{9}$ Women were chosen because the prevalence and incidence of depression is higher in this group. ${ }^{20} 28$ Exposure to risk factors may vary by gender group. ${ }^{29}$

\section{METHODS}

The study used qualitative techniques to analyse in-depth individual interviews.
The sample included women over 18 years old, who were born in South Asia or whose parents were born in South Asia. South Asia was defined as per Statistics Canada as India, Sri Lanka, Bangladesh, Pakistan and Nepal. Recruitment was from a community-based mental health agency, which provides services specifically to racialised communities in Toronto, Ontario, in 2010. Community workers referred participants to the study or participants answered an advertisement placed in the community agency. Participants were informed about researcher's ethnic background, occupation and credentials. Also a description of the purpose of the research was given with the study information materials. They were then approached face-to-face by the researchers and consented.

Those who were unable to read and speak in English were not included in this pilot study as there was no funding for interpreters. In order to increase the heterogeneity of the sample, we purposively recruited participants who had migrated from different countries, had differing marital status and belonged to different religious groups.

Depression was confirmed using GHQ-12 questionnaire. Those who scored more than 12 points (using the Likert scale) were invited for interview. Participants were interviewed alone at the community mental health agency offices. It provided them with an environment to speak freely and openly about their experiences. The aim was to get a rich description of the perceived causes of depression from the perspective of the respondents.

Interviews were conducted by the first author (SE), a South Asian immigrant woman, and lasted 45-60 min. A topic guide was used to direct the flow of the interviews. The structure of the interview was as follows: participants were first asked to explain their personal, family and household circumstances. Then they were invited to talk about their health, their understanding of causes of their depression, their daily lifestyles, employment, the nature of any social and economic difficulties and any social problems they faced. Finally, the participants were asked to comment on how they believe that those circumstances related to their mental health status. Interviews were taped and transcribed. All the transcripts were anonymised. Field notes were also made during the interview.

Informed consent was obtained prior to each of the interview.

Thematic content analysis with some elements of grounded theory ${ }^{30}$ was used to analyse the data. Data collection, coding and analysis were interrelated processes. The analyses of interview transcripts were begun after the first interview. We used a modified version similar to the three-step coding and analysis approach (open coding, axial coding and selective coding) introduced by the Strauss and Corbin. ${ }^{30}$ Each transcript was read at least twice and core concepts were identified. Codes were assigned for the selected texts. This was further developed by adding subthemes which were 
followed by the detailed coding. A preliminary coding scheme was developed after identifying the major themes. All three researchers have postdoctoral training in qualitative research and have published qualitative papers in peer-reviewed journals. The relationships and differences between codes were identified. Coding was done separately by each researcher (SE and KM) and then discussed by the research team. The researchers cross-checked their coding structures and, in cases where mismatches occurred, we conducted detailed discussions to achieve consensus. $\mathrm{NVivo}_{9}$ qualitative data analysis software ${ }^{31}$ was used for the data analysis. Recruitment was stopped when no new themes were being identified from the transcripts.

\section{RESULTS}

\section{The sample}

There were 10 participants in the study. Everyone who was referred took part. There were no dropouts. They were aged between 22 and 65 years. Seven women were born in India, two in Sri Lanka and one in Pakistan. Four women were Muslim, three Hindu and three Catholic. Two participants had university degrees, one had a high school diploma and seven had completed less than a high school education. None of the women were employed, and all were dependent on some form of income support from the government such as Ontario Disability Support Program, Employment Insurance or the Old Age Security program. Eight women were married, one was unmarried and the other was a widow. Two of the women had some history of mental illness other than depression.

\section{Causes of depression}

Three significant factors that cause depression were emerged from participant's narratives:

1. Individual, family and relationships,

2. Culture and migration and

3. Socioeconomic.

Participants endorsed a variety of combinations of these problems with some identifying difficulties in all three as the cause of their depression. Table 1 presents a summary of themes and subthemes emerged from the narratives.

\section{Individual, family and relationship factors}

Individual, family and relationship factors were identified as the primary cause of depression by the participants. Domestic abuse, infidelity, stress of divorce or separation and bereavement were the main stressors behind the depression.

\section{Abuse}

All members of the sample group experienced some form of domestic abuse such as physical, social, psychological, verbal or economic abuses from their intimate partners. Prolonged traumatic and abusive experiences were viewed as the root cause of their depression.

Financial or economic abuse was reported frequently by the participants. Seven women explained that they were forced to 'find jobs', 'work all 7 days' or that they were not allowed to have their own bank accounts. Three of our participants claimed that their husbands stole money from them, used their credit cards without authorisation and that they were forced to get bank loans.

Table 1 Thematic framework of the results

\section{Subthemes}

1. Individual, family and relationship factors Abuse

Financial/economic: controlling of income, forcing to work, stealing money, unauthorised use of credit cards, forcing to obtain loans.

Psychological: humiliation, threatening to take custody of children, restricting personal contacts, forcing not to talk to family and friends.

Physical: beating, slapping, pulling hair and choking.

Physical health problems

Multiple chronic illness, diabetes, high blood pressure, osteoporosis and fibromyalgia.

Bereavement

Lack of family support, difficulty in attending counselling, no social support networks in the neighbourhood.

Sexual infidelity

Ageing and isolation

Social and emotional isolation.

Difficulties in living with children's families, isolation, extra workloads such as child caring for grandchildren.

2. Culture and migration

Stress of divorce and separation

Negative response from family and community, cultural taboos on second marriage, loss of identity, fear and uncertainty of children.

Cultural distance

Parents: worry concerning westernizing of children, out of culture marriages.

Children: restricted family environments, strict rules, arranged marriages.

Labelling as a mentally ill, isolation from community

Stigma

Difficulties in new country

3. Socioeconomic factors

Economic difficulties

Loss of expectation, lack of understanding of systems, language difficulties.

Discrimination and racism

Lack of income from government income support programs, loss of jobs

Unfair treatments, racist acts, acts of hatred, discrimination for jobs 
ID 6 (45 years): "I had very stressful life. I worked in a factory. I used to work all 7 days. On Fridays my husband used to come to my work place. He took my cheque and asked me to sign the back of it. He takes all the money and gives me $\$ 20$ per week for the bus pass. I felt desperate. I had no money for anything... and he told to everybody: "I have a money maker at home"”

Restriction of personal contacts and demanding that women do not talk to other people in the neighbourhood were also identified as a main stressor. When husbands abandoned these women, they were devastated as they were socially isolated and left with little or no access to money. Lack of understanding of support systems, and fear of talking to unknown people, together with language difficulties, led these women to remain silent.

Physical abuse, such as beating, slapping and hair pulling, was mentioned by eight women in the study group. According to the participants, their children were also victims of these incidents. The other forms of psychological or physical abuse, such as being forced to get out of their home, being forced to wear hijab and being called names, were also mentioned.

\begin{abstract}
ID 4 (45 years): "In India it's perfectly normal for your husband to beat you...you have no choice other than just suffering. When I came here, I thought it's normal too and he hit me very badly to my shoulders, even blood came and my children cried. Neighbors also heard. I had this experience for 17 years."
\end{abstract}

\section{Health problems}

Seven of the participant's had been affected by some form of physical health problem. Narratives indicated that they felt overwhelmed by the stress of their poor health status. There were five participants, who were suffering from prolonged multiple illnesses, such as diabetes, high blood pressure, fibromyalgia and osteoporosis.

\begin{abstract}
ID 6 (45 years): "Sometimes I still I feel that why all these things happened to me. My whole body is paining. I was very active in the past... but now both hands are not good. I had an operation too. But still I can't even use my hand to eat food. Sometimes I keep plate on the table and eat like a dog."
\end{abstract}

\section{Bereavement}

According to ID 8 ( 54 years old and a widow with no children), bereavement was the main cause of her depression.

ID 8 (54 years): "He is a very good husband, he never leave me alone. Every time when I go to doctors appointment he comes with me. Now some times I don't eat, don't drink and crying all the time. Even I can't sleep. I remember him always. I got all this condition when he passed away."

Lack of extended family support, language difficulties that prevented her from attending grief counselling and an inability to develop supportive social networks in the neighbourhood made ID 8 more vulnerable to complicated grief reactions.

\section{Sexual infidelity}

The sexual infidelity of an intimate partner can be a devastating experience. Among the seven divorced or separated women in our sample, four mentioned that they discovered that their husbands were having extramarital relationships. They reported worry, sadness and depression following this.

\section{Ageing and isolation}

Two of the older-aged participants commented that their married children were not keen on caring for them, while others mentioned the burden of additional household chores such as caring for their grandchildren. Feelings of emotional and physical isolation, fear of the future because of their reliance on others and an inability to perform their own household activities (due to poor physical and mental health) led them to feel hopelessness and despair.

\section{Culture and migration}

\section{Stress of divorce and separation}

With the exception of one participant aged 33 years, none of the divorced or separated women were involved in secondary relationships. Thinking of a second marriage or having romantic relationships after marriage remains an unsuitable or out of culture concepts among the middle-aged and older women in this community. Results indicated that knowing that their former spouses had remarried or were currently involved in some sort of relationship brought these women more worry and jealousy.

Our samples claimed that traditionally in some South Asian cultures, divorce and separation are not acceptable and women may be blamed for a breakdown of their marriage. Participants painfully described the immense burden, stigma and stress experienced once they were divorced or separated from their spouses.

\begin{abstract}
ID 4 (45 years): "On the middle of the night he left me. I got a panic attack. I thought I am going to die. I was scared. How will society think now? What will happen to my children? How will my parents and my people react to me when I go to India? What am I going to tell them? When he started to get papers sent by the lawyer he phoned me and told me I am a characterless woman. That was first time I went to court, your family no body do that."
\end{abstract}

Participants also talked about the negative responses from their own family or other South Asian community members about their decision to get a divorce. Two of these women were still using their husband's surnames or lying about their actual family status, while others dealt with the problem by cutting themselves off from other South Asian people.

\section{Cultural distance}

Results showed that cultural distance between parent and children was also stressful for both groups. Those with strong South Asian values seemed to be more depressed and were not in a position to accept their children's out of culture marriages or pre-marital 
relationships. They further believed that they did not have power or authority to influence their children.

\begin{abstract}
ID 3 (65 years): "My son went out my culture and married. He married a Spanish girl. She doesn't want me to be in his life. Although I try hard to tell him she was not really kind of people we need he didn't listen to me. Now he is suffering. One day this woman asked me to make a beef soup. I don't eat or even touch beef. I really keep rules that I was taught to live."
\end{abstract}

In contrast, younger participants claimed they were suffering because of the strict rules of their parents. They identified themselves as being caught between traditional and modern. Two of our participants believed that they were brought up in more restrictive family environments than other Canadian girls in their age groups. Some said they had been asked to quit schools or choose jobs that are suited to girls or have an arranged marriage. Narratives further indicated that younger South Asian women were having more difficulty in negotiating their needs and expectations with their parents.

\section{Stigma}

The cultural stigma of being labelled as a mentally ill person was also a continuous stress factor. ID 2 (30 years) revealed her observation about the South Asian community and explained that some people believe that a person with a mental illness is an out control person or should be looked down upon or they should be scared to talk with them.

\section{ID 2: "My dad is saying that since you are mental, you should get married. And I said why? He said then your husband makes you happy. But if someone knows that I am a mentally ill, then they don't marry me. In our society there is a big label on mental illness."}

\section{Difficulties in new country}

Migration-related stress was also identified as a reason for depression. Migration is a transformation in immigrant's lives and it is associated with high hopes. Lack of understanding of the Canadian system, especially limited knowledge concerning the existing resources and language difficulties, made their lives more difficult and stressful.

\section{Socioeconomic factors \\ Economic difficulties}

Almost all the participants were living on form of government income support. Participants acknowledged that depression was often linked to financial difficulties, especially when there is insufficient income support or loss of employment.

\section{Discrimination and racism}

Subjective experiences with unfair treatment, racist acts or acts of hatred from other Canadians were also identified as a cause of depression and powerlessness. Poorer access to high standard jobs and differential treatment in health services and other service provision agencies were also highlighted. Participants openly talked about the challenges they faced in Canada, such as loss of cultural identity and loss of social pride. Muslim women acknowledged that wearing of a hijab caused some problems, especially after the $9 / 11$ terrorist attack in the USA. Participants also mentioned that immediate supervisors of other cultural backgrounds believed that people of South Asian ethnicity were inferior.

ID 4 (45 years): "Discrimination is $100 \%$ it's every where. Even at hospitals. If we ask something they take you easy. When people see me they think that I don't understand or speak English. Even at job. When I was working that lady gave me very hard time. The way you dress up. They don't like you. If you dress like their own dress, if you speak like them they will like you. But if you dress your own dress they will not happy."

\section{DISCUSSION}

The study findings highlight the multi-dimensional nature of the aetiological models of depression among South Asian immigrant women in Toronto, Canada.

Similar to studies in other countries, ${ }^{32-34}$ women of South Asian background in Canada perceived depression as an outcome of personal, family, cultural and social circumstances. Some of the stress factors, such as abuse, bereavement, sexual infidelity and the stress of divorce, were identified as the root causes of depression. Other stressors like physical health problems, ageing and isolation, cultural distance, stigma, difficulties faced in Canada, and socioeconomic problems were identified as continuous stressors but were considered less important and were less frequent.

The participants endorsed a personal social-cultural model for the aetiology of depression. In contrast with those previous study findings, none of our study participants offered spiritual, supernatural or religious causes of their depression. ${ }^{35}$

The majority of past studies on IEM of depression have used primary care patients with symptoms of depression $^{8} 33$ or general population samples. ${ }^{24}$ In contrast, our sample was of women who were being followed by a community mental health provider. It may be that our sample had more chronic or severe depression or were people who were more accepting of social and biological explanations and mental health system treatment.

The findings reveal the depth of violent and traumatic living experiences suffered by some women of South Asian origin. Some of their stories had remained secret for years. Such silence in the face of psychological trauma has been identified in other studies of women in other parts of the world. ${ }^{19}$ The association between recent life events and depression has been well documented. ${ }^{36-40}$ As has the association with long-term negative self-esteem, lack of social support, loss of intimate relationship, living alone and poor physical health conditions. $^{36} 4041$

The results of studies conducted with South Asian immigrant women in the UK are consistent with some of 
our findings. ${ }^{42} 43$ The belief that depression has social origins seems to be common in South Asian groups. Many women from these groups link their depression with losses such as bereavement, ill-health or job-related events. $^{43}$

Immigrants face many challenges in their new countries; however, such challenges can be more profound during the adjustment process for women with less education and power. ${ }^{36}{ }^{42}$ First-generation South Asian origin women have been reported to be more likely to have lower self-evaluation and self-esteem. ${ }^{14}$ The existing vulnerabilities and powerlessness of some South Asian women may increase after their arrival to Canada. ${ }^{44}$ The degree and nature of abuse and oppression faced by South Asian women in Canada has been well documented by the previous studies. ${ }^{45}{ }^{46}$ Our participants indicated that this was a major cause of depression. ${ }^{47}$

Divorce or separation is still considered unacceptable in many South Asian groups. ${ }^{48}$ Lack of traditional support systems to solve domestic violence and marriage disputes in immigrant groups is problematic. These are designed to solve marriage problems and keep the marriage together. ${ }^{23}{ }^{40}$ Lack of these led to some of the women in this study to get support from legal support services in Canada which led to divorce.

There is some evidence that people of South Asian origin in Canada maintain close ties with their country of origin and more strongly preserve their cultural heritage. ${ }^{27}$ This was confirmed in by the views of oldaged group. However, results further indicate that strong attachment to the culture of their own countries has created a cultural distance and conflict between parents and children. The cultural vulnerability faced by immigrants, specially by the South Asian immigrant women, has been identified as a main cause for depression by studies conducted in the UK. ${ }^{42} 49$

We acknowledge the fact that this is a small study. It was based in Toronto, and there may be differences in SA populations living in rural areas and different urban areas in Canada. The study was conducted in English, and this may have imposed some limitations on the findings as the participants may have felt more comfortable describing their perceived causes of depression in their own languages. The study also uses the term South Asian to identify a broad group of people with significant cultural, social and historical differences. It is of interest that despite this, their narratives and the causes they thought were the main reasons for their depression were similar.

We do not know how representative the results are. Indeed participants were selected from a communitybased mental health agency, and this inevitably introduces an element of sampling bias. It may be that we selected people who had more severe depression and we may also have selected people who were more likely to accept mainstream help. However, with regards to the last point, it is worth pointing out that the service we used to identify participants is a community-based agency which specifically offers help to racialised groups and is run by multi-cultural staff with a South Asian woman as the chief executive. The service is funded by Ontario but is not considered by many as mainstream. Those who did not speak English were excluded, and this may have skewed the demographic. Despite all of this, the narratives from our participants made the authors feel compelled to share the data. This study offers an in-depth and sometimes harrowing insight into the world of South Asian women in Toronto and the perceived causes of their depression. If these factors are the cause of depression for some in the community, then decreasing their impact may be a possible avenue that could be further investigated for the development of health promotion or illness prevention targeted at this population.

This pilot study used a qualitative approach. It demonstrated that recruitment of South Asian women with depression is possible that this group will discuss causation of depression and it has led to a model for the types of causes identified. It has been used to write a grant for a large-scale mixed methods approach that would allow quantification of the rates of particular risk factors and their associations and interactions.

\section{SUMIMARY}

In summary, this study provides an insight to conceptual models of depression among the South Asian origin women in Toronto, Canada. Attributions of depression heavily consisted of individual, family, relationships, social, cultural or economic consequences rather than biological or spiritual causation. The findings of this study further enhance our understanding of the social determinants of mental health and clearly show how individuals understand the combination of individual, family, social or environmental causation. Communities that perceive their illnesses as caused by social factors often prefer social remedies. Understanding the perceived causes of depression in the South Asian origin population of Toronto may help in the development of culturally appropriate prevention strategies.

Acknowledgements The authors would like to thank Across Boundaries, a community-based mental health centre, for their support during data collection.

Contributors All the authors designed and implemented the study. SE assisted with data collection. SE, FA and KM performed the statistical analysis. All authors contributed to drafts and approved the final draft of the manuscript. All authors had full access to the data in the study and can take responsibility for the integrity of the data and the accuracy of the data analysis.

Funding This research was supported by a strategic training grant (grant number TUF 96115) from the Canadian Institutes of Health Research provided for the Social Aetiology of Mental Illness Training Program.

\section{Competing interests None.}

Ethics approval Ethics approval was obtained from the Research Ethics Board of the Centre for Addiction and Mental Health in Toronto, Ontario, prior to the recruitment.

Provenance and peer review Not commissioned; externally peer reviewed. 
Data sharing statement Our qualitative data are not available to be shared as we did not consent patients for data sharing when the study was undertaken.

\section{REFERENCES}

1. Karasz A, Garcia N, Ferri L. Conceptual models of depression in primary care patients: a comparative study. J Cross Cult Psychol 2009;40:1041-59.

2. Kleinman A. Patients and Their Healers in the Context of Culture. Berkeley CA: University of California Press, 1980.

3. Kleinman A. The IIIness Narratives: Suffering, Healing and the Human Condition. New York: Basi Books Inc. Publishers, 1988.

4. Lloyd KR, Jacob KS, Patel V, et al. The development of the Short Explanatory Model Interview (SEMI) and its use among primary-care attender's with common mental disorders. Psychol Med 1998;28:1231-7

5. Goldberg D, Huxley P. Mental Illness in the Community: The Pathway to Psychiatric Care. London: Tavistock, 1980.

6. McCabe R, Priebe S. Explanatory models of illness in schizophrenia: comparison of four ethnic groups. Br J Psychiatry 2004;185:25-30.

7. Bhana K, Daniels CS. Generational changes in conceptions of mental illness: a study of a South African Indian sample. J Cross Cult Psychol 1986;17:493-504.

8. Brown C, Dunbar-Jacob J, Palenchar DR, et al. Primary care patients' personal illness models for depression: a preliminary investigation. Fam Pract 2001;18:314-20.

9. Lackey GF. Feeling blue in Spanish: a qualitative inquiry of depression among Mexican immigrants. Soc Sci Med 2008;67:228-37.

10. Sumathipala A, Hewege S, Hanwella R, et al. Randomized controlled trial of cognitive behaviour therapy for repeated consultations for medically unexplained complaints: a feasibility study in Sri Lanka. Psychol Med 2000;30:747-57.

11. Bhugra D, Mastrogianni A. Globalization and mental disorders. $\mathrm{Br} \mathrm{J}$ Psychiatry 2004;184:10-20.

12. Canadian Mental Health Association. Fast Facts: Mental Health/ Mental IIIness. http://www.cmha.ca/bins/content_page.asp?cid=620-23-43 (accessed 8 Apr 2011).

13. Health Canada. A proactive Approach to Good Health. Report to the Auditor General of Canada, Chapter 9. http://www.oag-bvg.gc.ca/ internet/English/parl_oag_200112_09_e_11830.html (accessed 5 Jun 2011).

14. Lai DW, Surood S. Predictors of depression in aging South Asian Canadian. J Cross Cult Gerontol 2008;23:57-75.

15. Whitley R, Kirmayer LJ, Groleau D. Understanding immigrants' reluctance to use mental health services: a qualitative study from Montreal. Can J Psychiatry 2006;51:205-9.

16. Kirmayer LJ, Weinfeld M, Burgos G, et al. Use of health care service for psychological distress by immigrants in and urban multicultural milieu. Can J Psychiatry 2007;52:295-304.

17. Beiser M, Simich L, Pandalangat N. Community in distress: mental health needs and help-seeking in the Tamil community in Toronto. Int Migrat 2003;41:233-45.

18. Simich L, Hamilton H, Baya BK. Mental distress, economic hardship and expectations of life in Canada among Sudanese newcomers. Transcult Psychiatry 2006;43:419-45.

19. Nduna M, Jewkes RK. Silence: a strategy used to deal with psychological distress by young people in the Eastern Cape, South Africa. Vulnerable Children and Youth Studies 2011;6:360-72.

20. Hammarstrom A, Lehti A, Danielsson U, et al. Gender-related explanatory models of depression: a critical evaluation of medical articles. Public Health 2009;123:689-93.

21. Goldstein B, Rosselli F. Etiological paradigms of depression: the relationship between perceived causes, empowerment, treatment preferences, and stigma. J Ment Health 2011;12:551-63.

22. Schulberg HC, McCelland MA. Conceptual model for educating primary care providers in the diagnosis and treatment of depression. Gen Hosp Psychiatry 1987;9:1-10.

23. Campbell LC, Clauw DJ, Keefe FJ. Persistent pain and depression a biopsychosocial perspective. Biol Psychiatry 2003;54:399-409.

24. Karasz A. Cultural differences in conceptual models of depression. Soc Sci Med 2005;60:1625-35.
25. Schreiber R, Hartrick GK. Keeping it together: how women use the biomedical explanatory model to manage the stigma of depression. Issues Ment Health Nurs 2002;23:91-105.

26. Statistics Canada. Ethnic Origin and Visible Minorities, Release no 72011. http://www12.statcan.gc.ca/census-recensement/2006/rt-td/ eth-eng.cfm (accessed 5 Dec 2010).

27. Tran K, Kaddatz J, Allard P. South Asians in Canada: Unity through diversity. Canadian Social Trends 2005;11:20-5.

28. Kuehner C. Gender differences in unipolar depression: an update of epidemiological findings and possible explanations. Acta Psychiatr Scand 2003;108:163-74.

29. Creed F, Winterbottom M, Tomenson B, et al. Preliminary study of non-psychotic disorders in people from the Indian subcontinent living in UK and India. Acta Psychiatr Scand 1999;99:257-60.

30. Strauss AL, Corbin JM. Basics of Qualitative Research; Techniques and Procedures for Developing Grounded Theory. London: Sage, 1998.

31. QSR International. NVivo Qualitative Data Analysis Software; QSR International Pty Ltd. Version 9. 2010. http://www.qsrinternational. com/support_faqs_detail.aspx?view $=11$

32. Bhui K, Bhugra D, Goldberg D. Causal explanations of distress and general practitioners' assessments of common mental disorder among punjabi and English attendees. Soc Psychiatry Psychiatr Epidemiol 2002;37:37-45.

33. Patel V, Pereira J, Mann AH. Somatic and psychological models of common mental disorder in primary care in India. Psychol Med 1998;28:135-43.

34. Ravi Shankar B, Saravanan B, Jacob KS. Explanatory models of common mental disorders among traditional healers and their patients in rural South India. Int J Soc Psychiatry 2006;52:221-33.

35. Sheikh S, Furnham A. A cross-cultural study of mental health beliefs and attitudes towards seeking professional help. Soc Psychiatry Psychiatr Epidemiol 2000;35:326-34.

36. Ahmad F, Shik A, Vanza R, et al. Voices of South Asian women: immigration and mental health. Women Health 2004;40:113-30.

37. Holmes $\mathrm{TH}$, Rahe $\mathrm{RH}$. The social readjustment rating scale. $J$ Psychosom Res 1967;11:213-18.

38. Jacob KS, Bhugra D, Lloyd KR, et al. Common mental disorders, explanatory models and consultation behaviour among Indian women living in the UK. J R Soc Med 1998;91:66-71.

39. Prince $\mathrm{M}$, Harwood $\mathrm{RH}$, Blizard RA, et al. Social support deficits, loneliness and life events as risk factors for depression in old age. The Gospel Oak Project VI. Psychol Med 1997;27:323-32.

40. Weich S, Lewis G. Poverty, unemployment and common mental disorders: population based cohort study. BMJ 1998;317:115-19.

41. Schulz A, Israel B, Williams D, et al. Social inequalities, stressors and self reported health status among African American and white women in the Detroit metropolitan area. Soc Sci Med 2000;51:1639-53.

42. Furnham A, Malik R. Cross-cultural beliefs about depression. Int Soc Psychiatry 1994;40:106-23.

43. Gask L, Aseem S, Waquas A, et al. Isolation, feeling 'stuck' and loss of control: understanding persistence of depression in British Pakistani Women. J Affect Disord 2011;128:49-55.

44. Morrison L, Guruge S, Snar KA. Sri Lankan Tamil immigrants in Toronto: gender, marriage patterns and sexuality. In: Kelson G, Delaureat B, eds. Gender, Immigration, and Policy. New York: New York University Press, 1999:144-60.

45. Ahmad F, Driver N, McNally MJ, et al. "Why doesn't she seek help for partner abuse?" An exploratory study with South Asian immigrant women. Soc Sci Med 2009;69:613-22.

46. Mason $\mathrm{R}$, Hyman I, Berman $\mathrm{H}$, et al. "Violence is an international language": Tamil women's perceptions of intimate partner violence. Violence Against Women 2008;14:1397-412.

47. Shackelford TK, Buss DM, Bennett K. Forgiveness or breakup: sex differences in responses to a partner's infidelity. Cogn Emot 2002;16:299-307.

48. Huang W. An Asian perspective on relationship and marriage education. Fam Process 2005;44:161-73

49. Hussain F, Cochrane R. Depression in South Asian women living in the UK: a review of the literature with implications for service provision. Transcult Psychiatry 2004;41:253-70. 